\title{
Inequalities and Approximations of Weighted Distributions by Lindley Reliability Measures, and the Lindley-Cox Model with Applications
}

\author{
Broderick O. Oluyede ${ }^{1}$, Macaulay Okwuokenye ${ }^{2}$ \& Karl E. Peace ${ }^{3}$ \\ ${ }^{1}$ Department of Mathematical Sciences, Georgia Southern University, Statesboro, GA 30460, USA \\ 2 Biogen Inc, Cambridge, MA 02142, USA \\ 3 Jiann-Ping Hsu College of Public Health, Georgia Southern University, Statesboro, GA 30460, USA \\ Correspondence: Broderick O. Oluyede, Department of Mathematical Sciences, Georgia Southern University, \\ Statesboro, GA 30460, USA. Tel: 1-912-478-5427. E-mail: boluyede@ georgiasouthern.edu
}

Received: January 31, 2015 Accepted: February 22, 2015 Online Published: March 31, 2015

doi:10.5539/ijsp.v4n2p61 URL: http://dx.doi.org/10.5539/ijsp.v4n2p61

\begin{abstract}
In this note, stochastic comparisons and results for weighted and Lindley models are presented. Approximation of weighted distributions via Lindley distribution in the class of increasing failure rate (IFR) and decreasing failure rate (DFR) weighted distributions with monotone weight functions are obtained including approximations via the length-biased Lindley distribution. Some useful bounds and moment-type inequality for weighted life distributions and applications are presented. Incorporation of covariates into Lindley model is considered and an application to illustrate the usefulness and applicability of the proposed Lindley-Cox model is given.
\end{abstract}

Keywords: Weight functions, Stochastic inequalities, Survival function, Ordering distributions, Bounds, LindleyCox Model, Covariates.

\section{Introduction}

Weighted distributions occur naturally in probability and statistics, and provides an approach to dealing with model specification and data interpretation problems. Applications of weighted distributions to biased samples can be seen in various areas including medicine, ecology, reliability, and branching processes. The reader can see the works of Patil and Rao (1978), Oluyede (2011) and references therein. When observations are selected with probability proportional to their length the resulting distribution is referred to as a length-biased distribution. Zelen and Feinleib (1969) introduced weighted distribution to discuss length biased sampling and showed that cases of chronic diseases identified by early detection screening programs constitute a length-biased sample due to the fact that individuals with a long pre-clinical disease phase have greater probability of being identified. Length-biased distributions are discussed by several authors including but not limited to Gupta and Keating (1985), Patil and Rao (1978). Oluyede (1999) developed and presented useful results on inequalities and selection of experiments for length-biased distributions. Additional results on exponential dominance and uncertainty for weighted residual life measures are given by Oluyede (2011).

We consider a non-negative random variable $X$ with its natural pdf $f(x ; \theta)$, where $\theta$ is a parameter. The pdf of the corresponding weighted random variable $X^{w}$ is given by:

$$
f^{w}(x ; \theta, \beta)=\frac{w(x, \beta) f(x ; \theta)}{\omega},
$$

where the weight function $w(x, \beta)$ is a non-negative function, that may depend on the parameter $\beta$, and $0<\omega=$ $E(w(X, \beta))<\infty$ is a normalizing constant.

In general, consider the weight function $w(x)$ defined as follows:

$$
w(x)=x^{k} e^{l x} F^{i}(x) \bar{F}^{j}(x) .
$$


Setting $k=0 ; k=j=i=0 ; l=i=j=0 ; k=l=i=0 ; k=l=j=0$ and $k=l=0, i \rightarrow i-1, j=n-i$ in this weight function, one at a time, implies probability weighted moments, moment-generating functions, moments, proportional hazards, proportional reversed hazards, and order statistics, respectively, where $F(x)=P(X \leq x)$ and $\bar{F}(x)=1-F(x)$. If $w(x)=x$, then $X^{*}=X^{w}$ is called the size-biased version of $X$.

Brown (1983) among others obtained measures of departure from exponentiality within the class of completely monotone distributions and the class of increasing mean residual life (IMRL) distribution functions. These measures of departure are given in terms of $\rho=\left|\frac{\mu_{2}}{2 \mu^{2}}-1\right|$, where $\mu_{2}=E\left(X^{2}\right)$ and $\mu=E(X)$. This is due to the fact that the exponential distribution satisfies $\rho=0$.

Keilson (1979) states that "If a system is modeled by a finite Markov chain which is ergodic, the passage from some specified initial distribution over the state space to a subset state space visited infrequently is often exponentially distributed to good approximation...... For engineering purposes, it is essential to quantify departure from exponentiality via error bounds. When one is dealing with time irreversible chains, e.g., systems with independent Markov components, the complete monotonicity present permits such quantification and the error bounds needed."

In this note, approximations via Lindley distribution in the class of weighted life distributions with monotone weight functions including distributions generated under proportional hazards transforms are obtained. This paper is organized as follows. Section 2 contains some preliminary concepts, weighted and Lindley distributions as well as stochastic comparisons. The connection between weighted distributions and distributions generated under proportional hazards transforms and results on stochastic dominance and comparisons are given. In section 3, bounds, inequalities and Lindley distributional approximations to weighted distributions are presented for the class of monotone failure rate distributions. Moment-type inequality for the comparison of weighted and Lindley distributions are also presented. Incorporation of covariates or concomitant information into Lindley model is considered and an application to illustrate the usefulness and applicability of the proposed Lindley-Cox model is presented in section 4 . Some concluding remarks are given in section 5.

\section{Stochastic Comparisons of Weighted and Lindley Distributions}

In this section, Lindley distribution (Lindley, 1958) as well as its generalizations, and weighted reliability function in general are presented. Let $\mathcal{F}$ be the set of absolutely continuous distribution function satisfying

$$
F(0)=0, \lim _{x \rightarrow \infty} F(x)=1, \operatorname{Sup}\{x: F(x)<1\}=\infty .
$$

Note that if the mean of a random variable with distribution function in $\mathcal{F}$ is finite, it is positive.

Now, let $X$ be a non-negative random variable with distribution function $F$ and reliability or survival function $\bar{F}$, where $\bar{F}=1-F$. We assume that $X$ is continuous with probability density function (pdf) $f$, where the reliability function $\bar{F}(x)=\int_{x}^{\infty} f(t) d t$. The weighted random variable $X_{W}$ has a reliability or survival function $\bar{F}_{W}(x)$ given by

$$
\bar{F}_{W}(x)=\frac{\bar{F}(x) E[W(X) \mid X>x]}{\delta^{*}},
$$

where $\delta^{*}$ is a normalizing constant. See Oluyede (2011) for details. The corresponding hazard or failure rate function is $h_{F_{W}}(x)=\frac{W(x) h_{F}(x)}{E[W(X) \mid X>x]}$, where $h_{F}(x)=f(x) / \bar{F}(x)$. The quantity $E[W(X) \mid X>x]$ is referred to as the weighted vitality function. Under the length-biased distribution, the weight function $W(x)=x$ and vitality function reduces to $E_{F_{l}}(X-x \mid X>x)+x=\delta_{F_{l}}(x)+x$, where $\delta_{F}(x)=\int_{x}^{\infty} \frac{\bar{F}(y) d y}{\bar{F}(x)}$ is the mean residual life function (MRLF). It is clear that if $V_{F_{l}}(x)=E_{F_{l}}[X \mid X>x]$ is increasing in $x$, then

$$
\overline{F_{l}}(x)=\mu^{-1} \int_{x}^{\infty} y d F(y) \geq c^{-1} \bar{F}(x) \geq \bar{F}(x),
$$

where $c=\bar{F}(0), \bar{F}_{W}(0)=\bar{F}_{l}(0)=1$ and $c^{-1}=\bar{F}_{l}(0) / \bar{F}(0)$. Indeed, if the weighted vitality function $E[W(X) \mid X>x]$ is increasing in $x$, then $\bar{F}_{W}(x) / \bar{F}(x)$ is increasing in $x \geq 0$.

\subsection{Lindley Distribution}

There are various generalizations of the Lindley distribution including those that were introduced by the following authors: Zakerzadeh and Dolati (2009), Nadarajah et al. (2011), Ghitany et al. (2013), Ghitany et al. (2008) and Oluyede and Yang (2014) among others. These generalizations constitute flexible family of distributions in terms 
of the varieties of shapes and hazard functions. The one parameter cdf of the Lindley distribution (Lindley, 1958) is given by

$$
F_{L}(x ; \theta)=1-\frac{1+\theta+\theta x}{1+\theta} e^{-\theta x}, \quad \text { for } x>0 \text {, and } \theta>0 .
$$

The corresponding Lindley pdf is given by

$$
f_{L}(x ; \theta)=\frac{\theta^{2}(1+x)}{1+\theta} e^{-\theta x}, \quad \text { for } x>0 \text {, and } \theta>0 .
$$

Lindley distribution is a mixture of exponential and gamma distributions, that is $f(x ; \theta)=(1-p) f_{G}(x ; \theta)+p f_{E}(x ; \theta)$ with $p=\frac{1}{1+\theta}$, where $f_{G}(x ; \theta) \equiv G A M(2, \theta)$, and $f_{E}(x ; \theta) \equiv E X P(\theta)$. The mean of Lindley distribution is given by $\mu=\frac{2+\theta}{\theta(1+\theta)}$.

A generalization of the Lindley distribution due to Zakerzadeh and Dolati (2009) is given as follows. Let $Y_{1}$ and $Y_{2}$ be two independently gamma distributed random variables with parameters $(\alpha, \theta)$ and $(\alpha+1, \theta)$ respectively. For $\gamma>0$, let $X=Y_{1}$ with probability $\frac{\theta}{\theta+\gamma}$ and $X=Y_{2}$ with probability $\frac{\gamma}{\theta+\gamma}$, then the pdf of $X$ (see Zakerzadeh and Dolati, (2009)) is given by

$$
f_{G L}(x ; \alpha, \theta, \gamma)=\frac{\theta^{2}(\theta x)^{\alpha-1} e^{-\theta x}}{(\theta+\gamma) \Gamma(\alpha)}+\frac{\gamma \theta(\theta x)^{\alpha} e^{-\theta x}}{(\theta+\gamma) \Gamma(\alpha+1)},
$$

or

$$
f_{G L}(x ; \alpha, \theta, \gamma)=\frac{\theta^{2}(\theta x)^{\alpha-1}(\alpha+\gamma x) e^{-\theta x}}{(\theta+\gamma) \Gamma(\alpha+1)},
$$

for $x>0, \theta>0, \alpha>0, \gamma>0$. Note that when $\alpha=\gamma=1$, we obtain the Lindley pdf given in equation (6). When $\gamma=0$ we have the gamma pdf with parameters $\alpha$ and $\theta$. If $\alpha=1$ and $\gamma=0$ the resulting pdf is the exponential pdf with parameter $\theta$. Additional generalizations of the Lindley distribution include work by Oluyede and Yang (2014), and Oluyede et al. (2015).

Suppose that the survival functions for the non-negative random variables $X$ and $Y$ with distribution functions $F$ and $G$ satisfy the proportional hazards model

$$
\bar{G}_{Y}(t)=\left[\bar{F}_{X}(t)\right]^{\beta}
$$

for all $t \geq 0$, and $\beta>0$; see Cox (1959) and Efron (1981) for details. Note that the survival function in equation (7) has the probability density function $g_{Y}(t)$ given by $g_{Y}(t)=\beta\left[\bar{F}_{X}(t)\right]^{\beta-1} f_{X}(t)$, for $\beta>0$, and $t \geq 0$. The weight function is given by $W(t) / \delta^{*}=\beta\left[\bar{F}_{X}(t)\right]^{\beta-1}$, for $t \geq 0$, and $\beta>0$. The corresponding failure rate function is given by $h_{Y}(t)=\beta h_{F}(t)$ for $t \geq 0$, and $\beta>0$. Under Lindley distribution, the density and hazard or failure rate functions, $g_{Y}(t)$ and $h_{Y}(t)$ are given by

$$
g_{Y}(t ; \theta, \beta)=\frac{\beta \theta^{2}(1+t)}{\theta+1}\left(1+\frac{\theta t}{1+\theta}\right)^{\beta-1} e^{-\beta \theta t}, \quad t>0, \theta>0, \text { and } \beta>0 .
$$

and

$$
h_{Y}(t ; \theta, \beta)=\frac{\beta \theta^{2}(1+t)}{1+\theta+\theta t}, \quad \text { for } t>0, \theta>0, \text { and } \beta>0,
$$

respectively.

\subsection{Some Relations and Stochastic Comparisons}

In this section, stochastic dominance and closure results for Lindley and weighted distributions as well as results on ordering of Lindley and weighted distributions and its implications on reliability measures are presented. First, some useful definitions and utility notions are given below.

Definition 2.1 Let $X$ and $Y$ be two non-negative random variables with distribution functions $F$ and $G$, respectively.

(i) The distribution functions $F$ and $G$ are stochastically ordered, denoted by $F<_{s t} G$, if $\bar{F}(x) \leq \bar{G}(x)$ for $x \geq 0$ (Shaked and Shanthikumar, 1994).

(ii) A distribution function $F$ is said to have increasing mean residual life (IMRL) on $(0, \infty)$ if $\mu_{F}=\int_{0}^{\infty} \bar{F}(x) d x<\infty$, $F(0)<1$ and $E(X-x \mid X>x)$ is increasing in $x \geq 0$.

(iii) A distribution function $F$ is said to have increasing (decreasing) failure rate for $x \geq 0$, denoted by IFR (DFR), if $F(0-)=0, F(0)<1$ and $P(X>x+t \mid X>t)=\bar{F}(x+t) / \bar{F}(t)$ is decreasing (increasing) in $t \geq 0$ for each $x>0$. 
(iv) Let $\mathrm{X}$ and $\mathrm{Y}$ be two non-negative random variables with probability density functions $f$ and $g$ respectively. The random variable $X$ is said to be larger than $Y$ in monotone likelihood ratio order $\left(X \geq_{l r} Y\right)$ if $f(x) / g(x)$ is non-decreasing in $x \geq 0$.

Note that if $F$ has DFR and $\mu_{F}=\int_{0}^{\infty} \bar{F}(x) d x<\infty$, then $F$ has IMRL. Note also that if the weight function $W(x)$ is increasing for $\geq 0$, then $X_{W} \geq_{l r} X$ and $h_{F_{W}}(x) \leq h_{F}(x)$ for all $x \geq 0$, so that $\bar{F}_{W}(x) \geq \bar{F}(x)$ for all $x \geq 0$, where $h_{F}(x)=f(x) / \bar{F}(x)$.

The next definition is due to Bhattacharjee (1986).

Definition 2.2 Let $X$ be in $\mathcal{F}$. The distribution function $F(x)$ of $X$ is said to be finitely and positively smooth if there exists a number $\alpha, 0<\alpha<\infty$, such that

$$
\lim _{t \rightarrow \infty} \bar{F}_{t}(x)=e^{-\alpha x}
$$

for all $x \geq 0$, where $\bar{F}_{t}(x)=P(X>x+t \mid X>t)=\frac{\bar{F}(x+t)}{\bar{F}(t)}$ is the survival function of a random variable $X_{t}$ (lifetime of a device at age $t, t \geq 0)$ and $\alpha$ is the asymptotic decay coefficient of $X$.

The next definition is due to Alzaid (1994).

Definition 2.3 Let $X$ be a non-negative random variable with distribution function $F$ in $\mathcal{F}$. If $F$ is finitely and positively smooth with asymptotic decay coefficient $\alpha$, then $X$ is called

(i) Used better than aged (UBA) if

$$
\bar{F}_{t}(x) \geq e^{-\alpha x}
$$

for all $t, x \geq 0$;

(ii) Harmonic used better than aged in expectation (HUBAE) if

$$
\int_{x}^{\infty} \bar{F}(t) d t \geq e^{-\alpha x} \int_{0}^{\infty} \bar{F}(y) d y
$$

provided $\mu=\int_{0}^{\infty} \bar{F}(y) d y<\infty$. The inequalities in (11) and (12) are reversed for used worse than aged (UWA) and harmonic used worse than aged in expectation (HUWAE).

In Theorems 2.1 and 2.2, we present useful ageing properties namely UBA and HUBAE for Lindley distribution as well as stochastic comparisons of proportional hazards Lindley distribution and weighted distributions with monotone weight function, respectively.

Theorem 2.1 (i) Lindley distribution function $F_{L}(x)$ is finitely and positively smooth with asymptotic decay coefficient $\theta$, (ii) $F_{L}(x)$ is Used better than aged $(U B A)$, and (iii) $F_{L}(x)$ is Harmonic used better than aged in expectation (HUBAE).

Proof. Note that

$$
\bar{F}_{L_{t}}(x)=\frac{\bar{F}_{L}(x+t)}{\bar{F}_{L}(t)}=\left(1+\frac{\theta x}{1+\theta+\theta t}\right) e^{-\theta x} .
$$

Now, it follows that $\lim _{t \rightarrow \infty} \bar{F}_{L_{t}}(x)=e^{-\theta x}$, for $0<\theta<\infty$.

(ii) Clearly,

$$
\bar{F}_{L_{t}}(x)=\left(1+\frac{\theta x}{1+\theta+\theta t}\right) e^{-\theta x} \geq e^{-\theta x},
$$

so that $F_{L}$ is UBA for all $t, x \geq 0$. 
(iii) Note that

$$
\begin{aligned}
\int_{x}^{\infty} \bar{F}_{L}(y) d y & =\int_{x}^{\infty}\left(1+\frac{\theta y}{1+\theta}\right) e^{-\theta y} d y \\
& =\frac{\theta+2}{\theta(\theta+1)} e^{-\theta x}+\frac{\theta x}{\theta(\theta+1)} e^{-\theta x} \\
& =\mu_{F_{L}} e^{-\theta x}+\frac{\theta x}{\theta(\theta+1)} e^{-\theta x} \\
& =e^{-\theta x} \int_{0}^{\infty} \bar{F}_{L}(y) d y+\frac{\theta x}{\theta(\theta+1)} e^{-\theta x} \\
& \geq e^{-\theta x} \int_{0}^{\infty} \bar{F}_{L}(y) d y
\end{aligned}
$$

where $\mu_{F_{L}}=\int_{0}^{\infty} \bar{F}_{L}(y) d y$. Consequently, $F_{L}$ of HUBAE.

Theorem 2.2 Let $\bar{F}_{W}(t)=\bar{G}_{Y}(t)=\left[\bar{F}_{L}(t)\right]^{\beta}, \beta>0$, where $F_{L}$ is the Lindley cdf, then for $t \geq 0,(i) h_{F_{W}}(t) \geq h_{F_{L}}(t)$ for $\beta \geq 1$, and $h_{F_{W}}(t)<h_{F_{L}}(t)$ for $0<\beta<1$, and $(i i) \bar{F}_{W}(t) \leq \bar{F}_{L}(t)$, for $\beta \geq 1$, and $\bar{F}_{W}(t)>\bar{F}_{L}(t)$, for $0<\beta<1$. (iii) If $F_{L}$ is an IFR (DFR) distribution and the weight function $W(t)$ is increasing (decreasing and convex), then $G_{Y}$ or $F_{W}$ is an IFR (DFR) distribution. (iv) $X_{W} \geq_{l r} X_{L}$.

Proof. The proof follows directly from the fact that $W(t) / \delta^{*}=\beta\left[\bar{F}_{L}(t)\right]^{\beta-1}$ is increasing in $t \geq 0$.

The next result deals with the closure of the HUBAE class of Lindley life distributions under proportional hazards transforms. The proof is straightforward and is omitted.

Theorem 2.3 Let $G_{Y_{i}}(x)$ be Lindley proportional hazards distributions that are HUBAE with equal asymptotic decay coefficient $\alpha$, then the distribution function $G_{Y}(x)=\sum_{i=1}^{\infty} p_{i} F_{Y_{i}}(x), 0 \leq p_{i} \leq 1, \sum_{i=1}^{\infty} p_{i}=1$, is HUBAE with asymptotic decay coefficient $\alpha$.

\section{Approximations via Lindley Distribution}

In this section, $L_{1}$ bounds for approximations via Lindley distribution to the class of weighted distributions including distributions generated under proportional hazards transforms are presented. Bounds and stability results on the proximity of the weighted distribution functions to the proportional hazards Lindley distributions as well as related distributions are established. These results are given in the context of distributions with monotone failure rate and mean residual life functions.

\section{$3.1 L_{1}$ Bounds and Approximations}

In this section, approximations of weighted reliability functions via Lindley distribution are presented. First we present the following useful result that is due to Barlow et al. (1963).

Theorem 3.1 If $F$ has DMRL, then $S_{k}(x) \leq S_{k}(0) e^{-x / \mu}, k=1,2, \ldots$, and $S_{k}(x) \geq \mu S_{k-1}(0) e^{-x / \mu}-\mu S_{k-1}(0)+$ $S_{k}(0), k=2,3, \ldots$, where

$$
S_{k}(x)= \begin{cases}\bar{F}(x) & \text { if } k=0, \\ \int_{0}^{\infty} \bar{F}(x+t) t^{k-1} d t /(k-1) ! & \text { if } k=1,2, \ldots,\end{cases}
$$

is a sequence of decreasing functions for which $F$ possess moments of order $J$, that is $\mu_{k}=E\left(X^{k}\right)$ exists, $k=$ $1,2, \ldots, J$.

We let $S_{-1}(x)=f(x)$ be the pdf of $F$ if it exists. Then $S_{k}(0)=\mu_{k} / k !$, and $S_{k}^{\prime}(x)=-S_{k-1}(x), k=0,1,2, \ldots, J$. The ratio $S_{k-1}(x) / S_{k}(x)$ is a hazard function of a distribution function with survival function $S_{k}(x) / S_{k}(0)$. The inequalities in Theorem 3.1 are reversed if $\mathrm{F}$ has increasing mean residual life (IMRL).

Theorem 3.2 If $\bar{F}_{W}(x)$ is an IFR survival or reliability function with a non-decreasing weight function, where $\bar{F}_{W}(x)$ is given by equation (4), then

$$
\int_{0}^{\infty}\left|\bar{F}_{W}(x)-\bar{G}_{Y}(x)\right| d x \leq 2 \mu\left|\beta^{-1}-\mu_{2} / 2 \mu^{2}\right|,
$$


where $\bar{G}_{Y}(x)=\left[\bar{F}_{X}(x)\right]^{\beta}, \bar{F}_{X}(x)=(1+x /(\mu+1)) e^{-x / \mu}$, for $x \geq 0$ and $0<\beta<1$.

Proof. Let $B=\left\{x \mid \bar{F}_{W}(x) \leq \bar{G}_{Y}(x)\right\}$. Then for $x \geq 0$, and $0<\beta<1$,

$$
\begin{aligned}
\int_{0}^{\infty}\left|\bar{F}_{W}(x)-\bar{G}_{Y}(x)\right| d x & =\int_{B}\left(\bar{G}_{Y}(x)-\bar{F}_{W}(x)\right) d x-\int_{B^{c}}\left(\bar{G}_{Y}(x)-\bar{F}_{W}(x)\right) d x \\
& \leq 2 \int_{B}\left(\bar{G}_{Y}(x)-\bar{F}_{W}(x)\right) d x \leq 2 \int_{B}\left(\bar{G}_{Y}(x)-\bar{F}(x)\right) d x \\
& \leq 2 \int_{0}^{\infty}\left(\bar{G}_{Y}(x)-\bar{F}(x)\right) d x \leq 2 \int_{0}^{\infty}\left(\bar{G}_{Y}(x)-S_{1}(x) / \mu\right) d x \\
& \leq 2 \int_{0}^{\infty}\left(e^{-\beta x / \mu}-S_{1}(x) / \mu\right) d x=2\left(\mu / \beta-S_{2}(0) / \mu\right) \\
& =2 \mu\left(\beta^{-1}-\mu_{2} / 2 \mu^{2}\right) .
\end{aligned}
$$

The first and third inequalities are straightforward, the second inequality follows from the fact that $W(x)$ is nondecreasing, so that $\bar{F}_{W}(x) \geq \bar{F}(x)$, for all $x \geq 0$. The fourth inequality follows from the fact that $(1+x /(\mu+$ 1) $)^{\beta} e^{-\beta x / \mu} \leq e^{-\beta x / \mu}$ for $0<\beta<1$. The fifth inequality follows directly by applying Theorem 3.1.

Corollary 3.1 Under the conditions of Theorem 3.2,

$$
\int_{0}^{\infty}\left|\bar{F}_{W}(x)-\bar{F}_{X}(x)\right| d x \leq 2 \mu \rho,
$$

where $\bar{F}_{X}(x)=e^{-x / \mu}$, for $x \geq 0$, and $\rho=\left|1-\mu_{2} / 2 \mu^{2}\right|$.

Theorem 3.3 If $\bar{F}_{W}^{*}(x)$ is an IFR survival or reliability function, with a non-decreasing weight function $W(x)>0$, then

$$
\int_{0}^{\infty}\left|\bar{F}_{W}^{*}(x)-(1+x /(\mu+1)) e^{-x / \mu}\right| d x \leq 2 \mu\left|1-\mu_{2} / 2 \mu^{2}\right|
$$

where $\bar{F}_{W}^{*}(x)=\frac{\bar{F}(x)\left(e^{h x}+M_{F}^{*}(x)\right)}{E_{F}\left(e^{h X}\right)}$, and $M_{F}^{*}(x)=\int_{x}^{\infty} \frac{\bar{F}(t) h e^{h t}}{\bar{F}(x)} d t$ for $x \geq 0$.

Proof. Let $\bar{F}^{*}(x)=(1+x /(\mu+1)) e^{-x / \mu}$ and $A=\left\{x \mid \bar{F}_{W}^{*}(x) \leq \bar{F}^{*}(x)\right\}$. Then for $x \geq 0$, we have

$$
\begin{aligned}
\int_{0}^{\infty}\left|\bar{F}_{W}^{*}(x)-\left(1+\frac{x}{\mu+1}\right) e^{-x / \mu}\right| d x & \leq \int_{0}^{\infty}\left|\bar{F}_{W}^{*}(x)-e^{-x / \mu}\right| d x \\
& \leq 2 \int_{A}\left(e^{-x / \mu}-\bar{F}_{W}^{*}(x)\right) d x \\
& \leq 2 \int_{0}^{\infty}\left(e^{-x / \mu}-\bar{F}(x)\right) d x \\
& \leq 2 \int_{0}^{\infty}\left(e^{-x / \mu}-S_{1}(x) / \mu\right) d x \\
& =2\left(\mu-S_{1}(0) / \mu\right)=2\left(\mu-\mu_{2} / 2 \mu\right) \\
& =2 \mu\left(1-\mu_{2} / 2 \mu^{2}\right) .
\end{aligned}
$$

The first inequality follows from the fact that $\left|\bar{F}_{W}^{*}(x)-(1+x /(\mu+1)) e^{-x / \mu}\right| \leq\left|\bar{F}_{W}^{*}(x)-e^{-x / \mu}\right|$. The second inequality is straightforward, the third inequality follows from the fact that $W(x)$ is non-decreasing, so that $\bar{F}_{W}^{*}$ and $\bar{F}$ are stochastically ordered. The fourth inequality follows from the application of Theorem 3.1.

Example 3.1 Consider the stationary renewal reliability function $\bar{F}_{W}$ corresponding to a reliability function $\bar{F}$, and given by

$$
\bar{F}_{W}(x)=\mu^{-1} \int_{x}^{\infty} \bar{F}(t) d t
$$

where $\mu=\int_{0}^{\infty} \bar{F}(t) d t$. Clearly, $\bar{F}_{W}(x)$ is absolutely continuous with failure rate function $h_{F_{W}}=\bar{F}(x) / \mu \bar{F}_{W}(x)=$ $(E[X-t \mid X>t])^{-1}$. The weight function is given by $W(x)=\left(h_{F}(x)\right)^{-1}$. Note that if $F$ is IMRL then $h_{F_{W}}(t)$ is decreasing. Now applying Theorem 3.3 with $\bar{F}_{W}^{*}=\bar{F}_{W}$, we have

$$
\int_{0}^{\infty}\left|\bar{F}_{W}^{*}(x)-e^{-x / \mu}\right| d x \leq 2 \mu\left|1-\mu_{2} / 2 \mu^{2}\right| .
$$


In particular, if

$$
\bar{F}(x ; \theta, \epsilon)= \begin{cases}e^{-(x-\theta) /(1-2 \epsilon)^{1 / 2}} & \text { if } x>\theta, \theta=1-(1-2 \epsilon)^{1 / 2} \\ 1 & \text { otherwise }\end{cases}
$$

then

$$
\int_{0}^{\infty}\left|\bar{F}_{W}^{*}(x)-e^{-(x-\theta) /(1-2 \epsilon)^{1 / 2}}\right| d x \leq 2 \mu\left|1-\mu_{2} / 2 \mu^{2}\right|=2 \epsilon
$$

Theorem 3.4 Let $\bar{F}_{W}^{*}(x)$ be a DFR survival or reliability function, with non-decreasing weight function $W(x)>0$. Then for $0<\beta \leq 1$,

$$
\int_{0}^{\infty}\left|\bar{F}_{W}^{*}(x)-\bar{G}_{Y}(x)\right| d x \geq 2 \mu^{2} e^{-c / \mu}\left|1-\frac{2 \mu+c+1}{\mu(\mu+1)}\right|,
$$

where $\bar{G}_{Y}(x ;, \mu, \beta)=\left[\bar{F}_{X}(x)\right]^{\beta}, \bar{F}_{X}(x)=\left(1+\frac{x}{\mu+1}\right) e^{-x / \mu}$, for $x \geq 0,0<\beta \leq 1, \mu>0$, and $c>0$.

Proof. Let $\bar{F}_{W}^{*}(x)$ be DHR. Then there exists $c>0$ such that $\bar{F}_{W}^{*}(x) \geq\left(1+\frac{x}{\mu+1}\right) e^{-x / \mu}$ or $\bar{F}_{W}^{*}(x) \leq\left(1+\frac{x}{\mu+1}\right) e^{-x / \mu}$ as $x \geq c$ or $x \leq c$. It follows therefore that for $0<\beta \leq 1$,

$$
\begin{aligned}
\int_{0}^{\infty}\left|\bar{F}_{W}^{*}(x)-\bar{G}_{Y}(x ; \mu, \beta)\right| d x & =2 \int_{c}^{\infty}\left(\bar{F}_{W}^{*}(x)-\left(1+\frac{x}{(\mu+1)}\right)^{\beta} e^{-\beta x / \mu}\right) d x \\
& \geq 2 \int_{c}^{\infty}\left(\bar{F}(x)-\left(1+\frac{x}{(\mu+1)}\right)^{\beta} e^{-\beta x / \mu}\right) d x \\
& \geq 2\left(\mu S_{1}(c)-\int_{c}^{\infty}\left(1+\frac{x}{(\mu+1)}\right)^{\beta} e^{-\beta x / \mu} d x\right) \\
& \geq 2 \mu\left(S_{1}(0) e^{\frac{-c}{\mu}}-\int_{c}^{\infty}\left(1+\frac{x}{(\mu+1)}\right) e^{\frac{-x}{\mu}} d x\right) \\
& =2 \mu^{2} e^{-c / \mu}\left(1-\frac{2 \mu+c+1}{\mu(\mu+1)}\right) .
\end{aligned}
$$

The first inequality is due to the fact that $W(x)$ is non-decreasing, so that $\bar{F}_{W}^{*}(x) \geq \bar{F}(x)$ for all $x \geq 0$ and the second and third inequalities follows from the fact that $0<\beta \leq 1$ and an application of Theorem 3.1.

Example 3.2 We apply the results of Theorem 3.4 to the stationary renewal reliability function $\bar{F}_{W}(x)=\mu^{-1} \int_{x}^{\infty} \bar{F}(t) d t$. Suppose $\bar{F}_{W}$ is a DFR reliability function, and

$$
\bar{F}(x ; \theta, \epsilon)= \begin{cases}e^{-(x-\theta) /(1-2 \epsilon)^{1 / 2}} & \text { if } x>\theta, \theta=1-(1-2 \epsilon)^{1 / 2} \\ 1 & \text { otherwise. }\end{cases}
$$

then by Theorem 3.4, we have

$$
\begin{aligned}
\int_{0}^{\infty}\left|\bar{F}_{W}^{*}(x)-\bar{F}_{X}(x ; \theta, \epsilon)\right| d x & \geq 2 \mu^{2} e^{-c / \mu}|1-(2 \mu+c+1) / \mu(\mu+1)| \\
& =2 e^{-c}|1-(2+c+1) / 2|=e^{-c}(c+1) .
\end{aligned}
$$

If $c=1$, then $\int_{0}^{\infty}\left|\bar{F}_{W}^{*}(x)-\bar{F}_{X}(x ; \theta, \epsilon)\right| d x \geq 2 e^{-1}$. Similarly, if $c=3$, then $\int_{0}^{\infty}\left|\bar{F}_{W}^{*}(x)-\bar{F}_{X}(x ; \theta, \epsilon)\right| d x \geq 4 e^{-3}$.

\subsection{Moment-Type Inequality}

In this section, we obtain bounds and moment-type inequality for the comparison of weighted distributions with Lindley distribution. We assume that the weighted distribution function $F_{W}$ is in $\mathcal{F}$, the set of absolutely continuous distribution functions given by equation (3).

The following results are due in part to the application of the lemma given by Brown (page 421,1983).

Theorem 3.5 Assume that $E\left(X^{k} \mid X>x\right)=\frac{\mu_{k} \bar{F}_{W}(x)}{\bar{F}(x)}$ is non-decreasing in $x \geq 0$,or $F_{W}$ has IMRL, and $0<\mu_{k}=$ $E_{F_{L}}\left[X^{k}\right]<\infty$, then for $k \geq 1$,

$$
\operatorname{Sup}_{x}\left|\bar{F}_{W}(x)-\bar{F}_{L}(x)\right| \leq 1-\mu_{k} / \mu_{F_{W}},
$$


where $\mu_{F_{W}}=\int_{0}^{\infty} \bar{F}_{W}(x) d x \geq \mu_{k}$, and $\bar{F}_{L}(x)$ is the Lindley reliability function.

Proof. Applying Brown's lemma and noting that $E\left(X^{k} \mid X>x\right)=\frac{\mu_{k} \bar{F}_{W}(x)}{\bar{F}(x)}$ is non-decreasing in $x \geq 0$, we obtain

$$
\begin{aligned}
\operatorname{Sup}_{x}\left|\bar{F}_{W}(x)-\bar{F}_{L}(x)\right| & \leq 1-\left(\mu_{k}\right)^{-1} \int_{0}^{\infty}\left[\mu_{k} / E\left(X^{k} \mid X>x\right)\right] x^{k} f_{L}(x) d x \\
& \leq 1-\left(\mu_{k}\right)^{-1} \mu_{F_{W}} \int_{0}^{\infty}\left(x^{k} f_{L}(x) / \mu_{F_{W}}\right)^{2} d x \\
& =1-\mu_{k} / \mu_{F_{W}} .
\end{aligned}
$$

Example 3.2 Keilson (1979) defines two first passage times, $T_{V}$ (the ergodic sojourn time) and $T_{E}$ (the ergodic exit time). The stationary renewal distribution for the finite state ergodic Markov process in continuous time is

$$
\bar{F}_{E}(x)=\mu_{V}^{-1} \int_{x}^{\infty} \bar{F}_{V}(t) d t,
$$

where $\mu_{V}=E\left(T_{V}\right)$. Consider a repairable three component parallel system with component failure rate 0.01 and component repair rate 1 . The time to first system failure is the first passage to a subset $B$ of the state space, where $B=\{(0,0,0)\}$. Brown (1983) showed that $E_{F_{E}}\left(T_{E}\right)=345,181.85$ and $E_{F_{V}}\left(T_{V}\right)=343,333.33$, so that

$$
\operatorname{Sup}_{x}\left|\bar{F}_{W}(x)-\bar{F}_{L}(x)\right| \leq 1-343,333.33 / 345,181.85=0.005355,
$$

where $\bar{F}_{W}=\bar{F}_{E}$ and $\bar{F}_{V}=\bar{F}_{L}$. Consequently, $F_{W}$ is within 0.005355 of $F_{L}$.

\section{The Lindley-Cox Model with Covariates}

In this section, the Lindley-Cox model and its application to real data is presented. Cox proportional hazard (PH) model (1972) is given by:

$$
h(t ; \mathbf{X}, \theta, \boldsymbol{\beta})=h_{0}(t ; \theta) \exp \left(\mathbf{X}^{\prime} \boldsymbol{\beta}\right),
$$

where $h_{0}(t ; \theta)$ is the baseline hazard function (of time only). Okwuokenye (2012) considered incorporation of covariate information using the Lindley distribution as a time-dependent component of the Cox's PH model. Using the Lindley model as a time-dependent component of Cox's PH model, the hazard rate and survival functions are

$$
h(t ; \mathbf{X}, \theta, \boldsymbol{\beta})=\frac{\theta^{2}(1+t)}{1+\theta+\theta t} \exp \left(\mathbf{X}^{\prime} \boldsymbol{\beta}\right),
$$

and

$$
\bar{F}_{L}(t ; \theta, \mathbf{X}, \boldsymbol{\beta})=\exp \left(-\exp \left(\mathbf{X}^{\prime} \boldsymbol{\beta}\right)(\theta t-\ln (1+\theta+\theta t)+\ln (1+\theta))\right),
$$

respectively. Okwuokenye (2012) called model resulting from (28) the Lindley-Cox model.

Suppose a time-to-event study (in which participants independently enter at different points) terminates at time T, the number of participants who will have the event by the termination point will be a random variable. Furthermore, suppose that the maximum time the $i^{\text {th }}$ participant may be observed is $T_{i}$, then $t_{i} \leq T_{i}, i=1,2, \ldots ., d$ denotes the observed times of events. However, $t_{r}, r=1,2, \ldots, c=n-d$ will be censored, representing the individuals who dropped out of the study, or were alive at the end of the study; that is, $t_{r} \geq T_{r}$. The likelihood of the sample consisting of $d$ complete and $n-d$ censored observations is:

$$
L(\theta, \boldsymbol{\beta})=\prod_{i=1}^{d} f\left(t_{i}, \mathbf{X}_{\mathbf{i}}, \theta, \boldsymbol{\beta}\right) \prod_{r=d+1}^{n} \bar{F}\left(T_{r}, \mathbf{X}_{\mathbf{r}}, \theta, \boldsymbol{\beta}\right) .
$$

The log-likelihood function is given by

$$
l(\theta, \boldsymbol{\beta})=\sum_{i=1}^{d}\left\{\ln \left(h\left(t_{i} ; \theta, \mathbf{X}, \boldsymbol{\beta}\right)\right)+\ln \left(\bar{F}\left(t_{i} ; \theta, \mathbf{X}, \boldsymbol{\beta}\right)\right)\right\}+\sum_{r=d+1}^{n} \ln \left(\bar{F}\left(T_{r} ; \mathbf{X}, \boldsymbol{\beta}\right)\right) .
$$


The LL function $L L=l(\theta, \boldsymbol{\beta})$ of a sample of $\mathrm{n}$ independent observations containing some right censored failure times is given by

$$
\begin{aligned}
L L & =2 n \ln (\theta)+\sum_{i=1}^{d} \ln \left(1+t_{i}\right)-\sum_{i=1}^{d} \ln \left(1+\theta+\theta t_{i}\right)+\sum_{i=1}^{d} \mathbf{X}_{\mathbf{i}}^{\prime} \boldsymbol{\beta} \\
& -\sum_{i=1}^{d}\left\{\exp \left(\mathbf{X}_{\mathbf{i}}^{\prime} \boldsymbol{\beta}\right)\left(\theta t_{i}-\ln \left(1+\theta+\theta t_{i}\right)+\ln (1+\theta)\right)\right\} \\
& -\sum_{r=d+1}^{n}\left\{\exp \left(\mathbf{X}_{\mathbf{r}}^{\prime} \boldsymbol{\beta}\right)\left(\theta t_{r}-\ln \left(1+\theta+\theta t_{r}\right)+\ln (1+\theta)\right)\right\},
\end{aligned}
$$

where $\mathbf{X}_{\mathbf{i}}=\left(X_{1}, \ldots, X_{p}\right)$ is a $p$-component vector of covariates and $\boldsymbol{\beta}$ is a $p \times 1$ vector of parameters.

\subsection{Lindley-Cox Model with Covariates: Applications}

Application of the Lindley-Cox model using a publicly available data set is presented in this section. The data set has been analyzed by other authors via different models. Lindley-Cox failure density is applied, to illustrate its application to the Fiegl and Zelen (1965) data. Fiegl and Zelen (1965) presented mortality data on two groups of patients, AG positive and AG negative, whose disease was diagnosed as acute myelogenous leukemia. Patients were classified as: AG positive if Auer rods, significant granulature of leukemia cells in the bone marrow, or both were present at the time of diagnosis; AG negative, if these conditions were absent. The data contained covariate information [white blood cell count (WBC)] and survival times in weeks. Survival times, times until death from time of leukemia diagnosis, were analyzed by the authors, in which they assumed that the expected survival time (the reciprocal of the parameter of the negative exponential failure density) is a linear function of the logarithm of covariate at the time of leukemia diagnosis. The model they assume is

$$
f\left(t_{i} ; \theta, x_{i}, \boldsymbol{\beta}\right)=\lambda_{i} \exp \left(-\lambda_{i} t_{i}\right), \quad \text { where } \lambda_{i}=\left(\alpha+\beta x_{i}\right)^{-1},
$$

and $x_{i}$ is the WBC for the $i^{\text {th }}$ patient. Model parameters was estimated iteratively using the maximum likelihood estimation technique. Peace (1976) assumed exponential failure density and used $\lambda_{i}=\theta_{0} \exp \left(\beta x_{i}\right)$ in his form of Cox's PH model. Estimation was done by expansion of the two equations of partial derivatives of the loglikelihood, with respect to the parameters, in a Taylor series to first order terms. They also estimated the hazard and survival functions, and performed a chi-square goodness-of-fit test to assess the fit of the model. We present results from using a Lindley-Cox model to analyze the data for the AG negative group. The parameter estimates are obtained by the maximum likelihood method. We give parameter estimates (see Tables 1, and 2) for assessing correlation between survival times and the covariate using the Feigl and Zelen model, Peace's negative exponential model, and Lindley-Cox model. The negative log-likelihood for the Feigl and Zelen model was not given by the authors; however, the negative log-likelihood for the Lindley-Cox model is slightly higher than that of the Peace's negative exponential model. Feigl and Zelen model and Lindley-Cox model give estimates of coefficient of covariate information that are negatively correlated with the survival times, while Peace's negative exponential gives the converse. The survival probability for the $i^{\text {th }}$ patient: $S_{i}=\exp \left(-\lambda_{i} t_{i}\right)$ for the Feigl and Zelen, and Peace negative exponential model.

For the Lindley-Cox model:

$$
S_{i}=\exp \left(-\exp \left(\mathbf{X}_{\mathbf{i}}^{\prime} \beta\right)(\theta t-\ln (1+\theta+\theta t)+\ln (1+\theta))\right) .
$$

Estimate for the survival distribution may be obtained by substituting the estimated values of the parameters in the respective equations. Estimates of the survival distributions, $\hat{S}_{F-}$ of Feigl and Zelen, $\hat{S}_{P-}$ of Peace for the $i^{\text {th }}$ patient in the AG negative group are

$$
\hat{S}_{F-}=\exp \left\{-\left(30-3 x_{i}\right)^{-1} t_{i}\right\}
$$

and

$$
\hat{S}_{P-}=\exp \left\{-0.0103 \exp \left(0.41138 x_{i}\right) t_{i}\right\}
$$

Estimate of the survival distributions $\hat{S}_{M_{-}}$of the Lindley-Cox model for the $i^{\text {th }}$ patient in the AG negative group is

$$
\begin{aligned}
\hat{S}_{M-} & =\exp \left\{-\exp \left(-0.2392 x_{i}\right)\left(0.2107 t_{i}-\ln \left(0.2107 t_{i}+0.2107+1\right)\right.\right. \\
& +\ln (0.2107+1))\} .
\end{aligned}
$$


Noted that $X_{i}$ is a component of the estimated function, so a value of $X_{i}$ (median in this case) that is, a representative of the covariate information is desired. The medians of the white blood cell count on the log scale is 4.30 for the AG negative group. Accordingly, the estimates of median survival distributions of the Feigl and Zelen, Peace negative exponential, and Lindley-Cox models for the AG negative group are

$$
\hat{S}_{F-}=\exp \left(-0.05847953 t_{i}\right), \quad \hat{S}_{P-}=\exp \left(-0.05864598 t_{i}\right)
$$

and

$$
\hat{S}_{M-}=\exp \left(-.3575214\left(0.2107 t_{i}-\ln \left(0.2107 t_{i}+1.2107\right)+0.191199\right)\right),
$$

respectively.

Table 1. Parameter Estimates and LL

\begin{tabular}{lcccc}
\hline Parameters & Group & F\&Z & Peace & Lindley-Cox \\
\hline$\hat{\theta}$ & $A G-$ & 30 & 0.0103 & 0.2107 \\
$\hat{\beta}$ & $A G-$ & -3 & 0.41138 & -0.2392 \\
\hline $\operatorname{var}(\hat{\theta})$ & $A G-$ & 1232.01 & $4.1 \times 10^{-7}$ & 0.03746 \\
$\operatorname{var}(\hat{\beta})$ & $A G-$ & 67.40 & 40.21779 & 0.08548 \\
$\operatorname{cov}(\theta, \beta)$ & $A G-$ & -284 & $-9.3804 \times 10^{-3}$ & $-5.533 \times 10^{-2}$ \\
\hline$-L L$ & $A G-$ & NG & 62.166 & 65.1046 \\
\hline
\end{tabular}

Table 2. Parameter Estimates and LL: Model with White Blood Cell

\begin{tabular}{lcc}
\hline Parameters & Lindley-Cox & Exponential \\
\hline$\hat{\theta}$ & 0.2107 & 0.0100 \\
$S E(\hat{\theta})$ & 0.1935 & 0.0161 \\
$\hat{\beta}$ & -0.2392 & 0.4114 \\
$S E(\hat{\beta})$ & 0.2924 & 0.3802 \\
\hline$h(t ; \hat{\theta}, w b c)$ & $\frac{\left(\hat{\theta}^{2}(1+\text { med.time })\right) e^{(\hat{\beta} m e d . w b c)}}{1+\hat{\theta}+\hat{\theta}(\text { med.time })}$ & $\hat{\theta} e^{(\hat{\beta}(\text { med.wbc }))}$ \\
& $\frac{\left((0.2107)^{2}(1+12)\right) e^{(4.3(-0.2392))}}{1+0.2107+0.2107(12)}$ & $0.0100 e^{(4.3(0.4114))}$ \\
& $=0.0552$ & $=0.0587$ \\
\hline$-L L$ & 65.1046 & 62.1656 \\
\hline$H_{0}: \beta=0$ vs & $-2 \ln \Lambda=0.6167$, & $-2 \ln \Lambda=1.1454$, \\
$H_{a}: \beta \neq 0$ & $p>0.05$ & $p>0.05$ \\
\hline
\end{tabular}

Note that $-2 \ln \Lambda \sim \chi_{1}^{2}$. Chi-square tests for goodness-of-fit were conducted using quartile intervals. Results for the chi-square tests reveal that we fail to reject the hypothesis that the Lindley-Cox provides a good fit to the AG negative group: $\chi^{2}=5.80$ with $p$-value $=0.055$. Based on the results, the Lindley-Cox model seems to provide a reasonable fit for AG negative group data.

\section{Concluding Remarks}

In this paper, stochastic orders, inequalities, bounds and relations for proportional hazards Lindley models and weighted life distributions in general are presented. Lindley distributional approximations and bounds are obtained for the class of IFR (DFR) weighted life distributions including proportional hazards models with monotone weight functions. Some useful results including moment-type inequality for the comparisons of weighted distributions with Lindley distribution are given. Covariate information is incorporated into the Lindley model to obtain results on Lindley-Cox model and applications are given to illustrate the usefulness of the developed results.

\section{References}

Alzaid, A. A. (1994). Aging Concepts for Items of Unknown Age. Communications in Statistics: Stochastic Models, 10, 649-659. http://dx.doi.org/10.1080/15326349408807314

Barlow, R., Marshall, A. W., \& Proschan, F. (1963). Properties of Probability Distributions with Monotone Hazard Rate. Annals of Mathematical Statistics, 34, 375-389. http://dx.doi.org/10.1214/aoms/1177703282 
Bhattacharjee, M. C. (1986). Tail Behavior of Age-Smooth Failure Distributions and Applications. Reliability and Quality Control (A. Basu, Ed.), Elsevier Science Publ.

Brown, M. (1983). Approximating IMRL Distributions by Exponential Distributions, with Applications to First Passage Times. Annals of Probability, 11, 419-427. http://dx.doi.org/10.1214/aop/1176993607

Cox, D. R. (1959). The Analysis of Exponentially Distributed Lifetimes with Two Types of Failures. Journal of the Royal Statistical Society, B21, 411-421.

Cox, D. R. (1972). Regression models and Life-tables. Journal of the Royal Statistical Society, Series B (Methodological), 34(2), 187-220.

Efron, B. (1981). Censored Data and Bootstrap. Journal of the American Statistical Association, 76, 312-319. http://dx.doi.org/10.1080/01621459.1981.10477650

Feigel, P., \& Zelen, M. (1965). Estimation of Exponential survival probabilities with Concomitant Information. Biometrics, 21, 826-838. http://dx.doi.org/10.2307/2528247

Gupta, R. C., \& Keating, J. P. (1985). Relations for Reliability Measures Under Length Biased Sampling. Scandinavian Journal of Statistics, 13, 49-56.

Ghitany, M. E., Al-Mutairi, D. K., Balakrishnan, N., \& Al-Enezi, L. J. (2013). Power Lindley Distribution and Associated Inference. Computational Statistics and Data Analysis, 64, 20-33. http://dx.doi.org/10.1016/j.csda.2013.02.026

Ghitany, M. E., Atieh, B., \& Nadarajah, S. (2008). Lindley Distribution and its Applications, Mathematics and Computers in Simulation, 78, 493-506. http://dx.doi.org/10.1016/j.matcom.2007.06.007

Keilson, J. (1979). Markov Chain Models - Rarity and Exponentiality, Springer-Verlag, New York.

Lindley, D. V. (1958). Fiducial Distribution and Bayes' Theorem. Journal of Royal Statistical Society, 20, 102-107.

Nadarajah, S., Bakouch, S. H., \& Tahmasbi, R. (2011). A Generalized Lindley Distribution, Sankhya B, 73, 331-359. http://dx.doi.org/10.1007/s13571-011-0025-9

Okwuokenye, M. (2012). Size and Power of Tests of Hypotheses on Parameters when. Modeling Time-to-Event Data with Lindley-Cox Distribution [Doctoral Dissertation], Jiann-Ping Hsu College of Public Health, Georgia Southern University.

Oluyede, B. O. (1999). On Inequalities and Selection of Experiments for Length-Biased Distributions. Probability in the Engineering and Informational Sciences, 13, 169-185. http://dx.doi.org/10.1017/s0269964899132030

Oluyede, B. O. (2011). Exponential Dominance and Uncertainty for Weighted Residual Life Measures. Probability in the Engineering and Informational Sciences, 25, 251-263. http://dx.doi.org/10.1017/s0269964810000380

Oluyede, B. O., \& Yang, T. (2014). A New Class of Generalized Lindley Distribution with Applications. Journal of Statistical Computation and Simulations, 1-29. http://dx.doi.org/10.1080/00949655.2014.917308

Oluyede, B. O., Yang, T., \& Omolo, B. (2015). A Generalized Class of Generalized Kumaraswamy Lindley Distribution with Applications to Lifetime Data. Journal of Computations and Modelling, 5(1), 27-70.

Patil, G. P., \& Rao, C. R. (1978). Weighted Distributions and Size-Biased Sampling with Applications to Wildlife and Human Families, Biometrics, 34, 179-189. http://dx.doi.org/10.2307/2530008

Peace, K. (1976). Maximum Likelihood Estimation and Efficiency Assessments of Tests of Hypotheses on Survival Probabilities, [Ph.D. Dissertation], Medical college of Virginia, Virginia Commonwealth University, Richmond, Virginia.

Shaked, M., \& Shanthikumar, J. G. (1994). Stochastic Orders and Their Applications, New York, Academic Press. http://dx.doi.org/10.1137/1037117

Zakerzadeh, H., \& Dolati, A. (2009). Generalized Lindley Distribution. Journal of Mathematical Extension, 3, 13-25.

Zelen, M., \& Feinleib, M. (1969). On the Theory of Chronic Diseases. Biometrika, 56, 601-614. http://dx.doi.org/10.1093/biomet/56.3.601 


\section{Copyrights}

Copyright for this article is retained by the author(s), with first publication rights granted to the journal.

This is an open-access article distributed under the terms and conditions of the Creative Commons Attribution license (http://creativecommons.org/licenses/by/3.0/). 